\title{
Homology modeling deduced tridimensional structure of Bacillus thuringiensis Cry1Ab18 toxin
}

\author{
Sudhanshu KashyaP ${ }^{1 *}$, Brahma Dev Singh ${ }^{2}$, DevindRa ViJay Amla ${ }^{3}$ \\ ${ }^{1}$ National Bureau of Agriculturally Important Microorganisms (ICAR), Kusmaur, Kaithauli, India \\ ${ }^{2}$ School of Biotechnology, Faculty of Science, Banaras Hindu University, Varanasi, India \\ ${ }^{3}$ Molecular Biology \& Genetic Engineering Division, National Botanical Research Institute, Lucknow, India \\ * Corresponding author: sudhanshukshyp@gmail.com
}

\begin{abstract}
Cry1Ab18 is an $\delta$-endotoxin produced by Bacillus thuringiensis strain. Till date the detailed mechanism of this toxin action is unclear. Therefore, solution of the three-dimensional structure of all Cry1 family members would be desirable for a comprehensive understanding of the initial mechanisms that underlie the toxicity of this type of toxin. Here, we predict a theoretical structural model of the newly reported Cry1Ab18 $\delta$-endotoxin, using

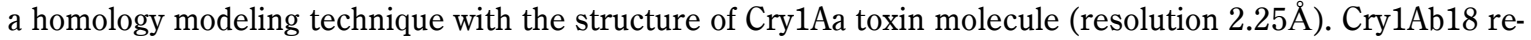
sembles Cry1Aa toxin by sharing a common three-domain structure. Domain I is composed of nine $\alpha$ helixes and one small $\beta$ strand, domain II is composed of nine $\beta$ strands and two $\alpha$ helixes and domain III consists of two $\alpha$ helixes and eleven $\beta$ strands. This model supports the existing hypotheses of receptor insertion and will further provide the initiation point for the domain swapping experiments aimed towards improving protein toxicity, and will help in the deeper understanding of the mechanism of action of common toxins.
\end{abstract}

Key words: three-dimensional structure, homology modeling, Cry1Ab18, $\delta$-endotoxin, Bacillus thuringiensis, 3-D model, MODELLER, predictive model, third party annotation

\section{Introduction}

During the last few decades, worldwide use of synthetic insecticides has caused problems of insect resistance and environmental pollution (Roush, 1996). The Insecticidal Crystal protein produced by the soil bacterium Bacillus thuringiensis belongs to a large toxin family with a target spectrum spanning agriculturally important insects, nematodes, protozoa and mammalian infesting flatworms (Schnepf et al., 1998; Roh et al., 2007). This family of proteins is widely used in biopesticide formulations and transgenic crops for insect control. Some of the factors that influence development of marketable formulations is a lower shelf life, evolution of insect resistance in targeted pests and a lower level of control of specific target pest. The mode of action for Cry1Ab toxins is a multistep process. First, the toxin must be ingested by the larvae; thereafter, it is solubilized and activated into an active form by the insect gut digestive fluids. Next, the activated toxin binds to specific proteins on the midgut miocrovilli. According to the most accepted model (Hofmann et al. 1988), solubilized monomeric toxin molecules bind to a receptor, further facilitating the initiation process necessary for toxin oligomerization. Toxin oligomerization shows high affinity binding to proteins attached to the cell membrane, mainly aminopeptidase or alkaline phosphatases. The inserted toxin disturbs the electrolyte balance by creating pores in the cell membrane, leading to cell lysis and finally to larval death (Knowles and Ellar, 1987). A detailed mechanism of toxin oligomerization and toxin oligomer insertion is difficult to establish. Therefore, solution of the threedimensional structure of all Cry1 family members is important for further investigations. So far, crystal structures of the active toxins in solutions have been analyzed for Cry3A (Li et al., 1991), Cry1Aa (Grochulski et al., 1995), Cry1Ac (Derbyshire et al., 2001), Cry3B (Galitsky et al., 2001), Cry2A protoxin (Morse et al., 2001), Cry4Ba (Boonserm et al., 2005), Cry4Aa (Boonserm et al., 2006) by X-ray diffraction crystallography, and subsequently Cry11Bb (Gutierrez et al., 2001), Cry5Ba 
1 YTPIDISLSLTQFLLSEFVPGAGFVLGLVDIIWGIFGPSQWDAFLVQIEQ 50

||||||||||||||||||||||||||||||||||||||||||||||||||

1 YTPIDISLSLTQFLLSEFVPGAGFVLGLVDI IWGIFGP SQWDAFLVQIEQ 50

51 LINQRIEEFARNQAISRLEGLSNLYQIYAESFREWEADPTNPALREEMRI 100

||||||||||||||||||||||||||||||||||||||||||||||||||

51 LINQRIEEFARNQAISRLEGLSNLYQIYAESFREWEADPTNPALREEMRI 100

101 QFNDMNSALTTAIPLLAVQNYQVPLLSVYVQAANLHLSVLRDVSVFGQRW 150

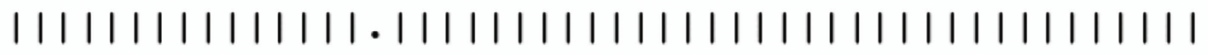

101 QFNDMNSALTTAIPLFAVQNYQVPLLSVYVQAANLHLSVLRDVSVFGQRW

150

151 GFDAATINSRYNDLTRLIGNYTDYAVRWYNTGLERVWGPDSRDWVRYNQF 200

||||||||||||||||||||||||||||||||||||||||||||$:|||| \mid$

151 GFDAATINSRYNDLTRLIGNYTDYAVRWYNTGLERVWGPDSRDWIRYNQF

200

201 RRELTLTVLDIVALFSNYDSRRYPIRTVSQLTREIYTNPVLENFDGSFRG

||||||||||||$:|| \cdot|||||\cdot||||||||||||||||||||||||||| \mid$

250

201 RRELTLTVLDIVSLFPNYDSRTYPIRTVSQLTREI YTNPVLENFDGSFRG

250

251 MAQRIEQNIRQPHLMDILNSITIYTDVHRGFNYWSGHQITASPVGFSGPE 300

$.|| .|| .:|| .|||||||:||||||| .|||\ldots||||||| .||||||||||$

251 SAQGIEGSIRSPHLMDILSS ITIYTDAHRGEYYWSGHQIMASPVGFSGPE

300

301 FAFPLFGNAGNAAPPV-LVSLTGLGIFRTLSSPLYRRI ILGSGPNNQELF $||||:.|\ldots||||| \ldots:|: \ldots| .|::||||| .|||\ldots:| .||||: \mid$.

301 FTFPLYGTMGNAAPQQRIVAQLGQGVYRTLSSTLYRPFNIGI--NNQQLS

349

348

350 VLDGTEFSFASLTTNLPST IYRQRGTVDSLDVIPPQDNSVPPRAGFSHRL

|||||| $\mid::$ : : : : ||||.:||: .|||||||.||||:|:||||.||||||

349 VLDGTEFAYGT-SSNLPSAVYRKSGTVDSLDE IPPQNNNVPPRQGFSHRL

400 SHVTMLSQ--AAGAVYTLRAPTFSWQHRSAEFNNI IPSSQITQIPLTKST

||$|:| \ldots \quad: \ldots:|\ldots:||| .||||||||||||||||||||||||||||$.

398 SHVSMFRSGFSNSSVSI IRAPMFSWIHRSAEFNNI IPSSQITQIPLTKST

447

448 NLGSGTSVVKGPGFTGGDILRRTSPGQISTLRVNITAPLSQRYRVRIRYA |||||||||||||||||||||||||||||||||||||||||||||||||| $\mid$ 448 NLGSGTSVVKGPGFTGGDILRRTSPGQISTLRVNI TAPLSQRYRVRIRYA

498 STTNLQFHTS IDGRPINQGNFSATMSSGSNLQSGSFRTVGFTTPFNFSNG

||||||||||||||||||||||||||||||||||||||||||||||||||

548 SSVFTLSAHVFNSGNEVYIDRIEFVPAEVT 577

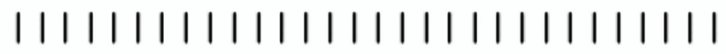

548 SSVFTLSAHVFNSGNEVYIDRIEFVPAEVT

577

Fig. 1. Depiction of the amino acid sequence alignment between Cry1Aa (upper row sequence) and Cry1Ab18 (lower row sequence) molecules. The similarity (92.2\%), gaps (1.0\%) and identity (88.1\%) between the sequences have been calculated with EBLOSUM62 matrix 
(Xia et al., 2008), and Cry5Aa (Min et al., 2009) have been predicted by the homology modeling method. These reports have shown that these toxins have three structural domains. Domain I is $\alpha$-helical bundle made of $7 \alpha$ helices. Domain II is composed of antiparallel beta sheets, and domain III is made up of $\beta$ sandwich. Here, we report the first monomeric toxin structure model for Cry1Ab18 toxin based on the hypothesis of its structural similarity with Cry1A toxin. This model supports the existing hypotheses of receptor insertion and will further provide the initiation point for the domain swapping experiments between Cry1 and other toxins thus elucidating the possible intermediate steps in the mode of toxin action.

\section{Methods}

The amino acid sequence of the putative Cry1Ab18 protein of Bacillus thuringiensis was retrieved from the GenBank using the sequence published earlier (NCBI: AAQ88259) (Stobdan et al., 2004). The core toxin protein was composed of 577 amino acids. A further attempt was made to find suitable template, using mGenTHREADER (http: cms.cs.ucl.sc.uk:3000/psipred), which is an online tool for searching similar sequences, based on the sequence and structure-wise similarity. From this homology search, Cry1Aa (PDB: 1CIY) was selected as a template protein. Finally, an amino acid sequence alignment between the target (Cry1Ab18) and the template protein was derived using MEGA4 software (Tamura et al., 2007). The three-dimensional structure of target protein was predicted by using the above alignment and running it with the python script file in MODELLER software (Sali et al., 1995). The resulted theoretical model was subjected to a series of tests for evaluating its consistency and reliability. Backbone confirmation was evaluated by the inspection of the Psi/Phi Ramachandran plot from RAMPAGE web server (http: mordred. bioc.cam.ac.uk). The energy criterion was evaluated with ProSA web server (http: prosa.services.came. sbg.ac.at) which compares the potential of mean forces derived from a large set of NMR and X-ray crystallographically derived protein structures of similar sizes. Potential deviations were calculated with SUPERPOSE web server (http: wishart.biology.ualberta.ca/cgi-bin/) for root mean square deviation (RMSD) between target and template protein structures. The visualization and refinement of the model was performed on UCF Chimera software (http: www. cgl.ucsf.edu/chimera) and PyMOL 0.99rc6 (http: pymol. org/funding.html). Figures and electrostatic potentials calculations were generated with PyMOL 0.99rc6.

\section{Results and discussion}

The Cry $1 \mathrm{Ab} \delta$ endotoxin is one of the many insecticidal toxins expressed by Bacillus thuriengiensis. The theoretical model of Cry1Ab18 toxin that was obtained corresponds to residues 70-647 of the primary structure (Fig. 1 and Fig. 2). The alignment of each domain was reliable due to clear correspondence of amino acids at $\mathrm{N}$-and $\mathrm{C}$ terminal sides, which can clearly define the end of the toxin molecule. The structural model shown in Fig. 3 indicates that it contains all the general features of Cry toxins (a $\alpha+\beta$ structure with three domains). The pore-forming domain I is composed of residues 72-327. It consists of 9 $\alpha$-helices and two small $\beta$-strands. The identified helices and strands are as follows: $\alpha 1$ ( $\left.\mathrm{Pro}^{72}-\mathrm{Ser}^{85}\right) ; \alpha 2\left(\mathrm{Ala}^{91}\right.$ Trp $\left.{ }^{102}\right) ; \alpha 3\left(\operatorname{Pro}^{107} \mathrm{Ile}^{121}\right) ; \alpha 4\left(\mathrm{Glu}^{127}-\mathrm{Ala}^{156}\right) ; \alpha 5\left(\mathrm{Pro}^{161}\right.$. $\left.\mathrm{Phe}^{185}\right) ; \alpha 6\left(\mathrm{Gln}^{191}-\mathrm{Trp}^{219}\right) ; \alpha 7\left(\mathrm{Ala}^{223}-\mathrm{Val}^{255}\right) ; \alpha 8\left(\mathrm{Ser}^{260}\right.$ $\left.\mathrm{Tyr}^{287}\right) ; \beta 0 \mathrm{a}\left(\mathrm{Ile}^{304}-\mathrm{Thr}^{306}\right) ; \alpha 9\left(\mathrm{Pro}^{308}-\mathrm{Asn}^{312}\right)$ and $\beta 0 \mathrm{~b}$ $\left(\mathrm{Ser}^{320}{ }_{-} \mathrm{Ser}^{327}\right)$. However, all the helices in the Cry1Ab18 model were slightly shorter than those in Cry1Aa. According to the amphiphilicity calculated with the Hoops and Woods values, the most exposed helices are $\alpha 1, \alpha 2 \mathrm{a}$, $\alpha 2 b, \alpha 3$ and $\alpha 6$, which corresponds well with the accessibility calculated with SwissPDB (http://spdbv.vitalit.ch/), except for $\alpha 1$, which is packed against domain II. Segura et al. (2000) expressed an opinion that this helix has some mobility and it has no apparent role in toxin activity once the protoxin part is cleaved off.

The toxins are released as protoxins which are solubilized in the midgut of insects and activated by gut proteases. Thus helix $\alpha 1$ probably does not play an important role in toxin activity after cleavage of the protoxin. It is assumed that the trigger for insertion of the pore-forming domain of the toxins into the epithelial cell membrane is a conformational change in the toxin, which occurs when another domain of the toxin binds to a receptor present on brush-border membranes (van Rie et al., 1990; Ahmad and Ellar, 1990). The model so far proposed for the organization of the pore-forming domain of the $B$. thuringiensis $\delta$-endotoxin within the membrane, is the Umbrella model (Li et al., 1991; Knowles, 1994; Gazit and Sahi 1995) which has been proposed previously for the colicin pore organization (Parker and 
$70-$,

YTPIDISLSLTQF L LSEFVPGAGFVLGLVDI IWG IF GPSQWDAF LVQIEQ

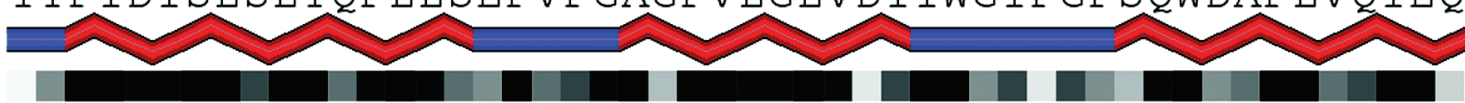

$120-169$

L INQR IEEFARNQA I SRLEGL SNLYQ I YAE SEREWEADP TNPALREEMR I

$170-219$

¿FNDMNSALTTA I PLFAVQNYQVPLLSVYVQAANLHLSVLRDVSVFGQRW

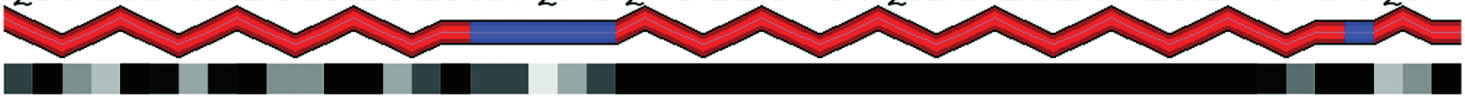

$220-1$ ' 269

GFDAAT INSRYNDL TRL I GNYT DYAVRWYNT GLERVWGPDSRDW I RYNQF

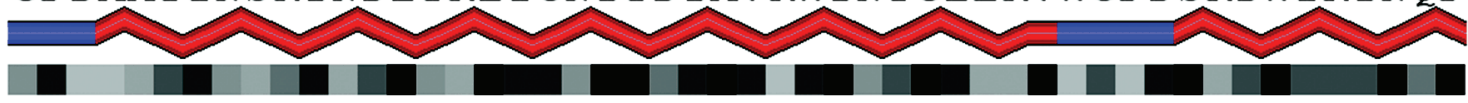

$270 \overline{\text { RELTLTVLDIVSLFPNYDSRTYPIRTVSQLTREIYTNPVLENFDGSFRG }}^{3} 19$

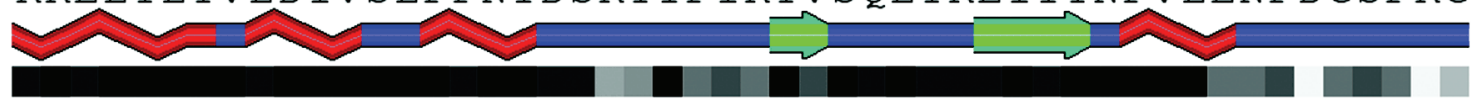

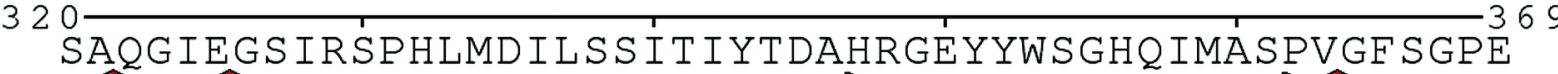

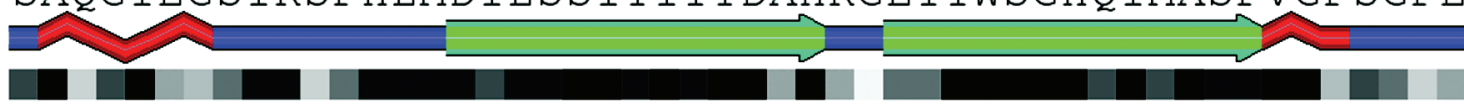

$370-419$

FTFPLYGTMGNAAPQQR IVAQLGQGVYRTLSSTLYRXPFN I G INNQQLSV

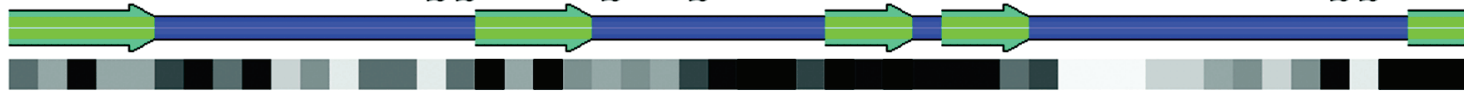

$420-4$, 469

LDGTEFAYGTSSNLPSAVYRKSGTVDSLDE IPPQNNNVPPRQGF SHRLSH

(

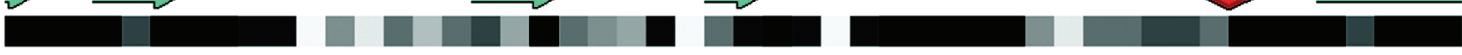

$470-519$

VSMFRSGF SNSSVS I IRAPMF SW I HRSAEFNN I IPSSQIT I PLTKS TNL (n)

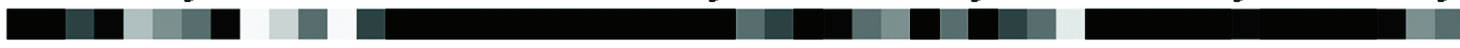

$520 \longrightarrow 569$

GSGT SVVKGPGF T GGD I LRRT SPGQ I ST LRVN I TAPL SQRYRVR IRYAS T

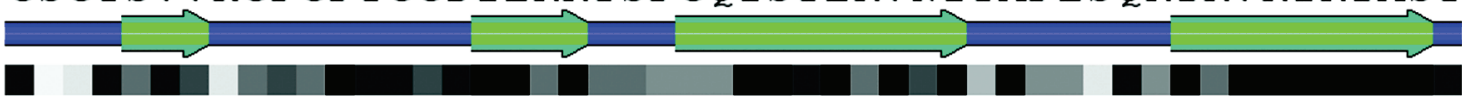

$570 \longrightarrow$, 619

TNLQFHTSIDGRP INQGNF SATMS SGSNLQSGSFRTVGFT TPFNF SNGSS

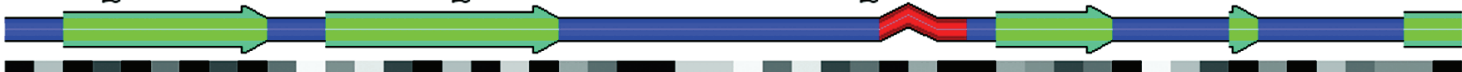

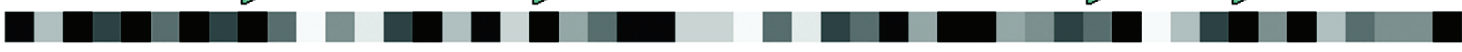

$620-647$

VFTLSAHVFNSGNEVYIDR IEFVPAEVT

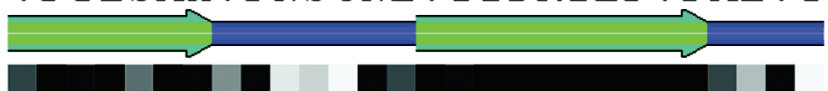

Fig. 2. The two dimensional structure annotation showing sequential arrangement of helices and sheets in Cry $1 \mathrm{Ab} 18$ toxin molecule. The structures designated are: $\approx$ helices; $\Longrightarrow$ E-beta-strand or bridge; $=$ C-coils; and shows Relative solvent accessibility (RSA) in a scale of 0 (completely buried,0-9\% RSA) to 9 (fully exposed, $90-100 \%$ RSA). The figure is generated using software polyview2D (http://polyview.cchmc.org/) 

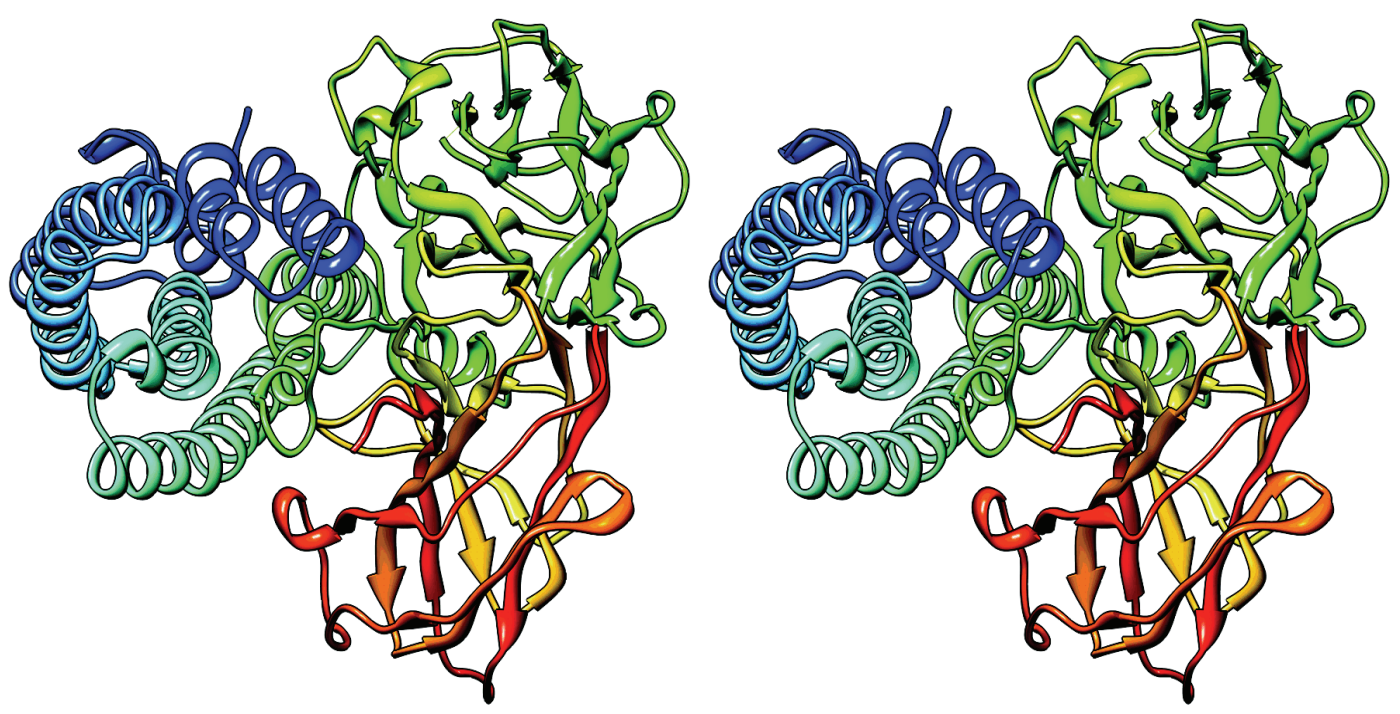

Fig. 3. Three-dimensional, three-domain structure of the Cry1Ab18 toxin oligomer. A complete molecular view from the top, showing a helical pore-forming domain and lower anti-parallel nature of sheets arrangement
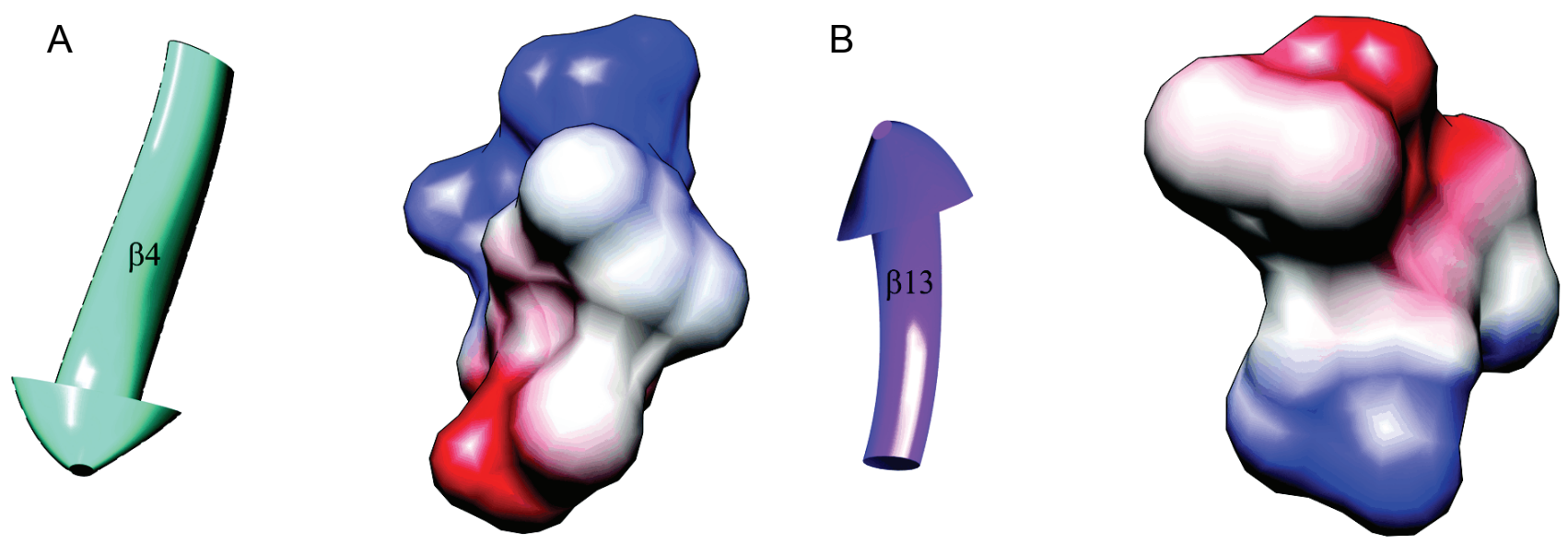

Fig. 4. The localized charge-distribution pattern along (A) $\beta 4$ and (B) $\beta 13$ showing a negative patch (blue) at their ends. The color scale is red $(+10)$ and blue $(-10)$

Table 1. The RMSD values between the reference structure of Cry1Aa1 and the generated model of Cry1Ab18

\begin{tabular}{l|c|c|c|c}
\hline \multicolumn{5}{c}{ Global \& Local RMSD Values } \\
\hline RMSD & Alpha carbons & Back bone & Heavy & All \\
\hline Atoms & 1.15 & 1.15 & 1.14 & 1.14 \\
\hline Structure & 574 & 2296 & 4428 & 4428 \\
\hline Cry1Aa & \multicolumn{4}{|c}{ Residues } \\
\hline Cry1Ab18 & \multicolumn{2}{|c}{$46-361,362-380,381-386,389-405,407-452,453-622$} \\
\hline
\end{tabular}

*The Global and local RMSD calculation was performed on SUPERPOSE server (average and pair wise) comparing the "per residue". Result values are in $\AA$ 
Pattus 1993). As it can be derived from the hypothesis that a pair of helices ( $\alpha 4$ and $\alpha 5$ ) joined on the side of domain I closest to the membrane surface would drop down into the membrane while the remaining helices will be rearranged to be open on the membrane surface like the ribs of an umbrella. Evidence based on voltage clamping experiments suggests that $\alpha 4$ and $\alpha 5$ have a structural role in the lining of the $\delta$-endotoxins pores in an umbrella-like structure. They have the ability of self- and co-assembling within the phospholipid membranes with their transmembrane orientation for their interactions between the membrane-bound helices (Gazit et al., 1998). The pore-forming properties of the toxins have also been demonstrated in studies in which activated $\delta$-endotoxins form single ion channels in planar lipid bilayers and cultured insect cells (Slatin et al., 1990; Schwartz et al., 1991).

The Cry1Ab18 domain I model relates well with the data from Gazzit et al. (1998), who suggested that $\alpha 4$ and $\alpha 5$ insert into the membrane in an antiparallel manner as a helical hairpin, and is in agreement with the hydrophobic hairpin hypothesis (Engelman and Steitz,

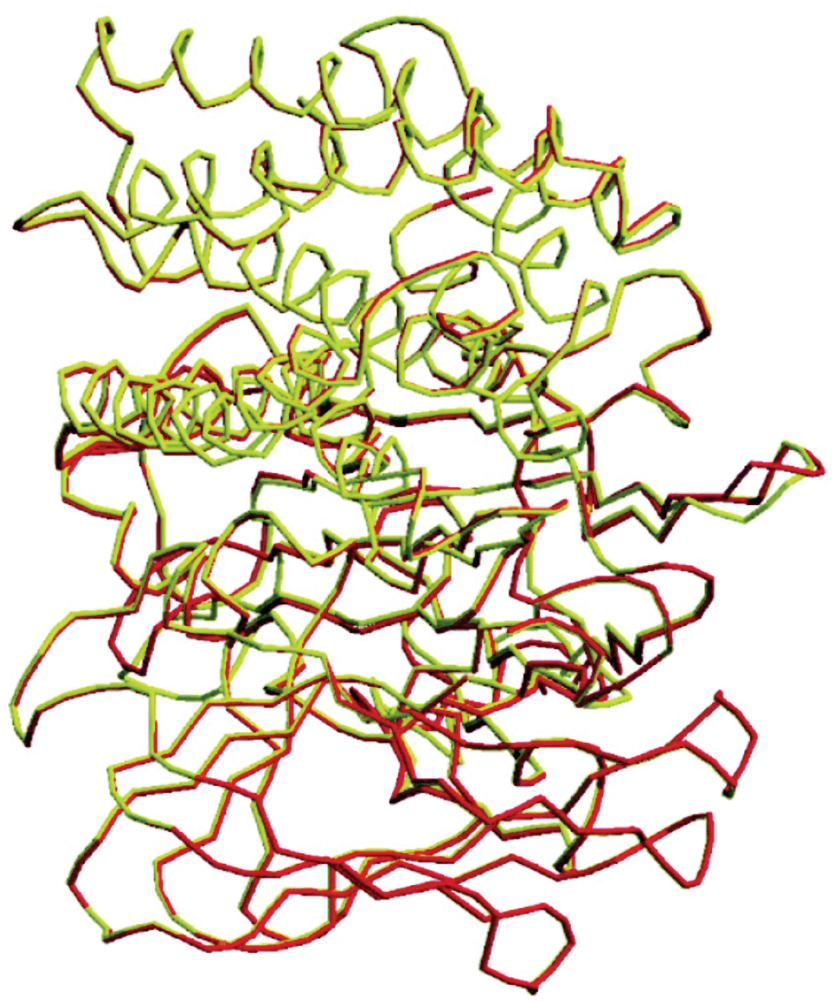

Fig. 5. Superpose backbone $3 d$ structure of Cry1Aa1 (yellow) and Cry1Ab18 (red) showing low structural deviations between the reference and the generated model
1981). The insertion of the $\alpha 4-\alpha 5$ hairpin into the membrane is also expected from theoretical considerations that the $C$ terminus of $\alpha 4$, the loop between $\alpha 4$ and $\alpha 5$, and the $\mathrm{N}$ terminus of $\alpha 5$ form a hairpin that contains the least polar segment of domain I (Grochulski et al., 1985), and the helices are joined on the side of the pore-forming domain proximal to the membrane. It is possible that according to the surface electrostatic potential of helices $\alpha 4$ and $\alpha 5$ and the presence of a neutral region in the middle of the helices, both helices cross the membrane with their polar sides exposed to the solvent. This notion has also been suggested by the results of mutagenesis experiments of Kumar and Aronson (1999) with Cry1Ac toxin. The structure of domain I of the toxin, the effect of site-directed mutagenesis in this domain on toxin activity, and studies with hybrid toxins (Ge et al., 1989; Ahmad and Ellar, 1990; Wu and Aronson, 1992) all suggest that domain I, or parts of it, inserts into the membrane and forms a pore. This idea is further supported by studies that show that truncated proteins corresponding to domain I of CryIA(c) (Walter et al., 1993) $\delta$-endotoxin form ion channels in model lipid membranes similar to those formed by the intact toxins.

Extensive mutagenesis studies indicate that mutations in $\alpha 5$, but not $\alpha 2$ or $\alpha 6$ helices result in synthesis of a substantial number of inactive or low-activity toxins (Wu and Aronson, 1992; Aronson et al., 1995). Studies with synthetic peptides corresponding to $\alpha 5$ and $\alpha 7$, the most conserved helices of the pore-forming domain from CryIIIA (Gazit and Shai, 1993; Gazit et al., 1994; Gazit and Shai, 1995) and $\alpha 5$ of CryIA(c) (Cummings et al., 1994) suggest that $\alpha 5$, but not $\alpha 7$, aggregates within lipid membranes, permeates phospholipid vesicles, and forms ion channels within planar lipid bilayers. Similar to the results obtained with model membranes, it was found that $\alpha 5$ binds insect midgut membranes, is protected from enzymatic proteolysis upon binding, and is cytotoxic to insect cells (Parker and Pattus, 1993). This region is also the most conserved among Cry toxins. Kumar and Aronson (1999) also demonstrated that mutations in the base of helix $\alpha 3$ and the loop between $\alpha 3$ and $\alpha 4$ that cause alterations on the balance of negative charged residues may cause toxicity loss. Mutations in helices $\alpha 2, \alpha 6$ and the surface residues of $\alpha 3$ have no important effect on toxicity; meanwhile, helices $\alpha 4$ and $\alpha 5$ seem to be very sensitive to mutations. It is possible that mutations aimed to an increase in amphilicity in 
A
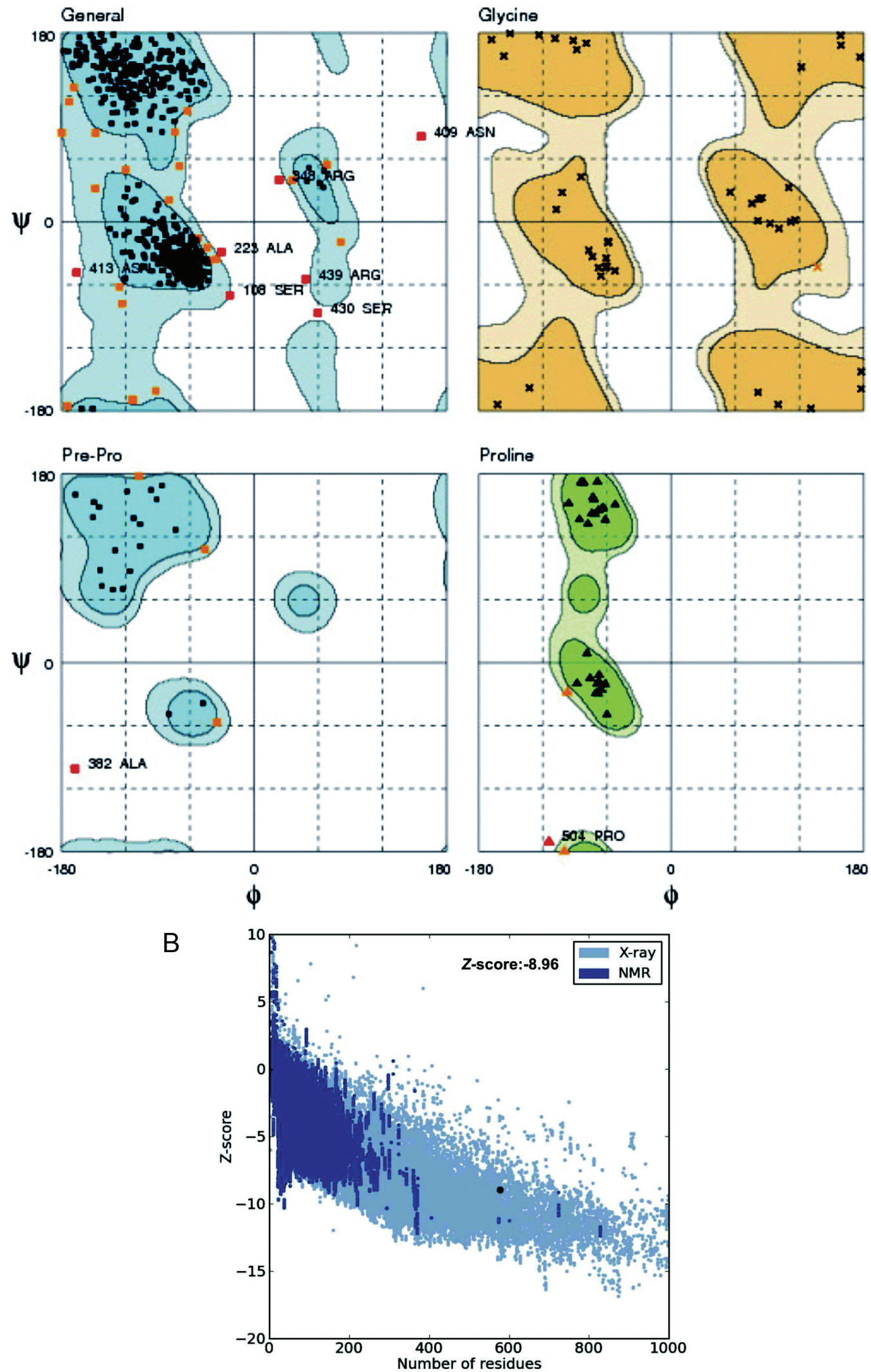

Fig. 6. (a) Ramachandran plot analysis showing placement of residues in the deduced model. Structure orientation residues have been separately considered for angle and torsions. General plot statistics are: residues in most favorable regions 535 (93.4\%); residues in additional allowed regions 29 (5.1\%); residues in disallowed regions 9 (1.6\%). Other plots are evaluated for specific residues as indicated at the top left corner of each plot. (b) Model validation of Cry1Ab18 with ProSA. The result shows that the structure has characteristic of native structures. The $Z$-score of -8.92 is highlighted with a large dot 
these helices will improve the pore-forming activity of Cry1Ab18 type of toxins. The other helices spread on the surface of the membrane.

After receptor binding, the network of contacts between $\alpha 7$, the helix in the interface between the poreforming domain and the receptor-binding domain, and $\alpha 5, \alpha 6$ and, presumably, $\alpha 4$ helices may assist the insertion of the $\alpha 4-\alpha 5$ hairpin into the membrane by the unpacking of the helical bundle that exists in the non-membrane-bound form of the toxin. This hypothesis might account for the observation that $\alpha 7$ mutants are susceptible to proteolysis by either trypsin or midgut juice (Dean et al., 1996). Our model also supports the notion that the $\alpha 4-\alpha 5$ hairpin is the major structural component in the lining of the pores formed by $\delta$-endotoxin. Therefore, it is possible to create toxin variants with better membrane permeability potential by stabilizing the hairpin antiparallel structure by cross-linking $\alpha 4$ with $\alpha 5$. This postulation is important because mutations within transmembrane segments of proteins usually decrease or have no effect on the biological activities of these proteins. Thus, it is conceivable that the introduction of several salt bridges or other bonds between $\alpha 4-\alpha 5$ helices, or the stabilization of the $\alpha 4-\alpha 5$ hairpin by the creation of bridging interactions between the $\alpha 3-\alpha 4$ and $\alpha 5$ $\alpha 6$ loops may result in a significantly enhanced toxic activity. Other studies also support the umbrella-like model for domain I insertion into membranes (Gazit et al., 1997; Masson et al., 1999; Schwartz et al., 1997b).

The charge distribution pattern in the Cry1Ab18 theoretical model corresponds to a negatively charged patch along $\beta 4$ and $\beta 13$ (Fig. 4a and $4 \mathrm{~b}$ ) of domains II and III, respectively. This is the most variable domain among Cry toxins, and it has been shown that it is involved in receptor recognition and therefore considered as the specificity determining region. As for other Cry toxins, domain II, the receptor-binding domain, is composed of three $\beta$-sheets with loops at the apex of the $\beta$-hairpin extensions, while Cry1Ab18 toxin consists of three Greek key beta sheets arranged in a beta prism topology. It is comprised of residues 336-494, two helix $\left(\alpha 10 \mathrm{Ser}^{446}-\mathrm{Glu}^{449}, \alpha 11 \mathrm{Pro}^{459}-\mathrm{Gly}^{462}\right)$ and $10 \beta$-strands ( $\beta 2$ $\mathrm{Ile}^{336}-\mathrm{His}^{347}, \beta 3 \mathrm{Glu}^{350}-\mathrm{Ser}^{361}, \beta 4 \mathrm{Arg}^{386}{ }^{3{ }^{3}{ }^{389}}{ }^{389}, \beta 5 \mathrm{Tyr}^{396}$ $\mathrm{Tyr}^{404}, \beta 6 \mathrm{Ser}^{418}{ }^{-\mathrm{Leu}^{426}}, \beta 7 \mathrm{Thr}^{436}-\mathrm{Ala}^{438}, \beta 8 \mathrm{Ala}^{443}-\mathrm{Tyr}^{445}$, $\left.\beta 9 \mathrm{His}^{465}-\mathrm{Phe}^{473}, \beta 10 \mathrm{Ala}^{487}-\mathrm{His}^{494}\right)$. Mutations in defined regions of the Cry1Aa toxin have been identified as essential for binding to the membrane of midgut cells of
Bombyx mori (Ge et al., 1989; Lu et al., 1994). In the Cry $1 \mathrm{Ab} 18$ model, this region is slightly longer than its counterparts in Cry1Aa. The loop between $\beta 2-\beta 3$ also seems to be able to modulate the toxicity and specificity (Smith and Ellar, 1994).

Domain III is comprised of residues 509-643, has a two antiparallel-sheet sandwich structure and shows highly conserved residues with the only important modification being a 3-residue deletion between $\beta 16$ and $\beta 17$. The $\beta$-strands in this domain are $\beta 12\left(\mathrm{Thr}^{589}{ }^{5 r o}{ }^{512}\right)$, $\beta 13\left(\mathrm{Thr}^{517}-\mathrm{Leu}^{519}\right), \beta 14\left(\mathrm{Ser}^{524}-\mathrm{Val}^{526}\right), \beta 15\left(\mathrm{Tle}^{536}-\mathrm{Arg}^{539}\right)$, $\beta 16\left(\mathrm{Gly}^{543}-\mathrm{Asn}^{551}\right), \beta 17\left(\mathrm{Tyr}^{560}-\mathrm{Ser}^{568}\right), \beta 18\left(\mathrm{Leu}^{572}-\mathrm{Ile}^{578}\right)$, $\beta 19\left(\mathrm{Arg}^{581}-\mathrm{Phe}^{588}\right), \alpha 12\left(\mathrm{Ser}^{600}-\mathrm{Ser}^{602}\right), \beta 20\left(\mathrm{Arg}^{604}-\mathrm{Gly}^{607}\right)$, $\beta 21\left(\mathrm{Ser}^{618}\right.$-His $\left.^{626}\right), \beta 22\left(\mathrm{Val}^{634}-\mathrm{Pro}^{643}\right)$. Several studies indicate that site mutations in this domain reduce toxicity and alter channel properties (Li et al., 199; Chen et al. 1993; Schwartz et al. 1997a). One consequence of the umbrella-like model of insertion would be the approach of the $\beta 17$ strand. An increase or decrease in the number of positive charges near the pore mouth would presumably affect pore conductance. The structural alterations in pore-forming region have an indirect affect on the ion channel function in the case of toxin molecules (Aronson et al. 1999). Collective results suggest that the prime role of this conserved $\beta 17$ strand region is the structural stability of the toxin.

Finally, the recognition of artifacts and errors in experimental and theoretical structures remains a problem in the field of structure modeling. A structural comparison of Cry1Aa, toxin with the theoretical model of the Cry $1 \mathrm{Ab} 18$ protein indicates correspondence with the general model for a Cry protein (Fig. 5). The superimposed backbone traces show low RMS deviations (Table 1). The Ramachandran plot indicates that most of the residues (93.4\%) have $\varphi$ and $\Psi$ angles in the favorable core orientations and $5.1 \%$ are in allowed regions (Fig. $6 a)$. Most bond lengths, bond angles and torsion angles are in the range of the values expected for a naturally folded protein. Web-based software tools like ProSA are diagnostic tools that are based on the statistical analysis of all available protein structures and are widely used for analysing the 3D models of protein structures for potential errors. Their range of application includes error recognition in experimentally determined structures, theoretical models and protein engineering (Wiederstein and Sippl, 2007). The software evaluates the model by parsing its coordinates and energy using a distance- 
based pair potential (Sippl, 1990; Sippl, 1995) and capturing the solvent exposed protein residues (Sippl, 1990; Sippl, 1995). The results are displayed in the form of a $Z$-score and a plot of residues energy. The $Z$-score shows the overall model quality and provides deviations from the random conformation (Sippl, 1993; Sippl, 1995). The plot checks whether the $Z$-score of the protein is within the range of similar proteins (NMR and X-ray derived structures). Groups of structures from different sources (X-ray, NMR) are distinguished by different colors as shown in Fig. $6 \mathrm{~b}$. The value of 8.92 is among the native conformation and the overall residues energy remains largely negative.

In conclusion, the evidence presented herein, based on the identification of structural equivalent residues of Cry1Aa in Cry1Ab18 toxin through homology modeling, indicate that due to high homology between these two toxins, they share a common tridimensional structure. Cry1Ab18 contains the most variable regions in the loops of domain II, which determine the specificity of these toxins. Taken together, our results are consistent with the umbrella-like model for the structure of the pores formed by a toxin. This is the first model of a Cry1Ab18 protein and its importance can be foreseen from a biotechnological perspective since the mutations or changes that abolish the pre-packing of the non-membrane-bound toxin could lead to the design of improved and more potent $\delta$-endotoxins.

\section{Acknowledgements}

The authors are grateful to the Indian Council of Agricultural Research, New Delhi, India for RAship to SK in the Network Project on "Application of Microorganisms in Agriculture and Allied Sectors". We are also thankful for the infrastructure facility and encouragement received from the Director of $\mathrm{Na}$ tional Bureau of Agriculturally Important Microorganisms.

\section{References}

Ahmad W., Ellar D.J. (1990) Directed mutagenesis of selected regions of a Bacillus thuringiensis entomocidal protein. FEMS Microbiol. Lett. 68: 97-104.

Aronson A.I., Geng C., Wu L. (1999) Aggregation of Bacillus thuringiensis Cry1A toxins upon binding to target insect larval midgut vesicles. AEM 65: 2503-2507.

Aronson A.I., Wu D., Zhang C. (1995) Mutagenesis of specificity and toxicity regions of a Bacillus thuringiensis protoxin gene. J. Bacteriol. 177: 4059-4065.

Boonserm P., Mo M., Angsuthanasombat C., Lescar J. (2006) Structure of the functional form of the Mosquito larvicidal
Cry4Aatoxin from Bacillus thuringiensis at a 2.8-angstrom resolution. J. Bacteriol. 188: 3391-3401.

Boonserm P., Davis P., Ellar D.J., Li J. (2005) Crystal structure of the Mosquito-larvicidal toxin Cry4Ba and its biological implications. J. Mol. Biol. 348: 363-382.

Chen X.J., Lee M.K., Dean D.H. (1993) Site-directed mutations in a highly conserved region of Bacillus thurngiensis $\delta$-endotoxin affect inhibition of short circuit current across Bombyx mori midgets. Proc. Natl. Acad. Sci. USA. 90: 9041-9045.

Cummings C.E., Arrmstrong G., Hodgmann T.C., Ellar D.J. (1994) Structural and functional studies of a synthetic peptide mimicking a proposed membrane inserting region of a Bacillus thuringiensis delta-endotoxin. Mol. Membr. Biol. 11: 87-92.

Dean D.H., Rajamohan F., Lee M.K., Wu S.-J., Chen X.J., Alcantara E., Hussain S.R. (1996) Probing the mechanism of action of Bacillus thuringiensis insecticidal proteins by sitedirected mutagenesis-a minireview. Gene 179: 111-117.

Derbyshire D.J., Ellar D.J., Li J. (2001) Crystallization of the Bacillus thuringiensis toxin Cry1Ac and its complex with the receptor ligand $N$-acetyl-D-galactosamine. Acta Crystallogr. Sect. D. 57: 1938-1944.

Engelman D.M., Steitz T.A. (1981) The spontaneous insertion of proteins into and across membranes: the helical hairpin hypothesis. Cell 23: 411-422.

Galitsky N., Cody V., Wojtczak A., Ghosh D., Luft J.R., Pangborn W., English L. (2001) Structure of the insecticidal bacterial endotoxin Cry3Bb1 of Bacillus thuringiensis. Acta Crystallogr. Sect D. 57: 1101-1109.

Gazit E., Bach D., Kerr I.D., Sansom M.S., Chejanovsky N., Shai Y. (1994) The alpha-5 segment of Bacillus thuringiensis delta-endotoxin: in vitro activity, ion channel formation and molecular modelling. Biochem. J. 304: 895-902.

Gazit E., Burshtein N., Ellar D.J., Sawyer T., Shai Y. (1997) Bacillus thuringiensis cytolytic toxin associates specifically with its synthetic helices $A$ and $C$ in the membrane bound state. Implications for the assembly of oligomeric transmembrane pores. Biochemistry 36: 15546-15554.

Gazit E., La Rocca P., Sansom M.S.P., Shai Y. (1998) The structure and organization within the membrane of the helices composing the pore forming domain of Bacillus thuringiensis $\delta$-endotoxin are consistent with an "umbrella-like" structure of the pore. Proc. Natl. Acad. Sci. USA 95: 12289-12294.

Gazit E., Shai Y. (1993) Structural and functional organization of the a 5 segment of Bacillus thuringiensis endotoxins. Biochemistry 32: 3429-3436.

Gazit E., Shai Y. (1995) The assembly and organization of the alpha 5 and alpha 7 helices from the pore-forming domain of Bacillus thuringiensis delta-endotoxin. Relevance to a functional model. J. Biol. Chem. 270: 2571-2578. 
Ge A.Z., Sivarova N.D., Dean D.H. (1989) Location of the Bombyx mori specificity domain of Bacillus thuringiensis $\delta$-endotoxin protein. Proc. Natl. Acad. Sci. USA 86: 4037-4041.

Grochulski P., Masson L., Borisova S., Pusztai-Carey M., Schwartz J.L., Brousseau R., Cygler M. (1995) Bacillus thuringiensis CryIA(a) insecticidal toxin: crystal structure and channel formation. J. Mol. Biol. 254: 447-464.

Gutierrez P., Alzate O., Orduz S.A. (2001) Theoretical model of the tridimensional structure of Bacillus thuringiensis subsp. medellin Cry 11Bb toxin deduced by homology modeling. Mem. Inst. Oswaldo Cruz. 96: 357-364.

Hofmann C., Vanderbruggen H., Hofte H., Van Mellaert H. (1988) Specificity of Bacillus thuringiensis delta-endotoxins is correlated with the presence of high-affinity binding sites in the brush border membrane of target insect midgets. Proc. Natl. Acad. Sci. USA 85: 7844-7848.

Knowles B.H., Ellar D.J. (1987) Colloid osmotic lysis is a general feature of the mechanism of action of Bacillus thuringiensis delta-endotoxins with different insect specificities. Biochim. Biophys. Acta 924: 509-518.

Knowles B.H. (1994) Mechanism of action of Bacillus thuringiensis insecticidal $\delta$-endotoxins. Adv. Insect. Physiol. 24: 275-308.

Kumar A.S.M., Aronson A.I. (1999) Analysis of mutations in the pore-forming region essential for insecticidal activity of a Bacillus thuringiensis $\delta$-endotoxin. J. Bacteriol. 181: 6103-6107.

Li J., Carroll J., Ellar D.J. (1991) Crystal structures of insecticidal $\delta$-endotoxin from Bacillus thuringiensis at 2.5 A resolutions. Nature 353: 815-821.

Lu H., Rajamohan F., Dean D.H. (1994) Identification of amino acid residues of Bacillus thuringiensis $\delta$-endotoxin CryIA (a) associated with membrane binding and toxicity to Bombyx mori. J. Bacteriol. 176: 5554-5559.

Masson L., Tabashnik B.E., Liu Y.B., Btousseau R., Schwartz J.L. (1999) Helix 4 of the Bacillus thuringiensis Cry1Aa toxin lines the lumen of the ion channel. J. Biol. Chem. 274: 31996-32000.

Min Z.X., Qui X.L., Zhi D.X., Xiang W.F. (2009) The theoretical three-dimensionalstructure ofBacillus thuringiensis Cry5Aa and its biological implications. Protein J. 28: 104-110.

Morse R.J., Yamamoto T., Stroud R.M. (2001) Structure of Cry2Aa suggests an unexpected receptor binding epitope. Structure 9: 409-417.

Parker M.W., Pattus F. (1993) Rendering a membrane protein soluble in water: a common packing motif in bacterial protein toxins. Trends Biochem. Sci. 18: 391-395.

Roh J.Y., Choi J.Y., Li M.S., Jin B.R., Je Y.H. (2007) Bacillus thuringiensis as a specific, safe, and effective tool for insect pest control. Microbiol. Biotechnol. 17: 547-559.

Roush R.T. (1996) Can we slow adaptation by pests to insect transgenic crops? In: Biotechnology and Integrated pest management, ed. Parsley G.J., CAB International, Wallingford, UK.

Sali A., Potterton L., Yuan F., van Vlijmen H., Karplus M. (1995) Evaluation of comparative protein modeling by MODELLER. Proteins. 23: 318-326.

Schnepf E., Crickmore N., van Rie J., Lereclus D., Baum J., Feitelson J., Zeigler D.R., Dean D.H. (1998) Bacillus thuringiensis and its pesticidal Crystal proteins. Microbiol. Mol. Biol. Rev. 62: 772-806.

Schwartz J.L., Garneau L., Masson L., Brousseau R. (1991) Early response of cultured lepidopteran cells to exposure to delta-endotoxin from Bacillus thuringiensis: involvement of calcium and anionic channels. Biochim. Biophys. Acta 1065: 250-260.

Schwartz J.-L., Juteau M., Grochulski P., Cygler M., Préfontaine G., Brousseau R., Masson L. (1997b) Restriction of intramolecular movements within the Cry1Aa toxin molecule of Bacillus thuringiensis through disulfide bond engineering. FEBS Lett. 410: 397-402.

Schwartz J.L., Potvin L., Chen X.J., Brousseau R., Laprade R., Dean D.H. (1997a) Single-site mutations in the conserved alternating-arginine region affect ion channels formed by CryIAa a Bacillus thuringiensis toxin. Appl. Environ. Microbiol. 63: 3978-3984.

Segura C., Guzman F., Patarroyo M.E., Orduz S. (2000) Activation pattern and toxicity of the Cry $1 A b 18 B b$ toxin of Bacillus thuringiensis subsp. Medellin. J. Invertebr. Pathol. 76: $56-62$.

Sippl M.J. (1990) Calculation of conformational ensembles from potentials of mean force: An approach to the knowledge-based prediction of local structures in globular proteins. J. Mol. Biol. 213: 859-883.

Sippl M.J. (1993) Recognition of errors in three dimensional structures of proteins. Protein Struct. Funct. Genet., 17: 355-362.

Sippl M.J. (1995) Knowledge-based potentials for proteins. Curr. Opin. Struct. Biol. 5: 229-235.

Slatin S.L., Abrams C.K., English L. (1990) Delta-endotoxins form cation-selective channels in planar lipid bilayers. Biochem. Biophys. Res. Commun. 169: 765-772.

Smith G.P., Ellar D.J. (1994) Mutagenesis of two surface exposed loops of the Bacillus thuringiensis CryIC $\delta$-endotoxin affects insecticidal specificity. Biochem. J. 302: 611616.

Stobdan T., Kaur S., Singh A. (2004) Cloning and nucleotide sequence of a novel cry gene from Bacillus thuringiensis. Biotech. Lett. 26: 1153-1156.

Tamura K., Dudley J., Nei M., Kumar S. (2007) MEGA4: Molecular Evolutionary Genetics Analysis (MEGA) software version 4.0. Mol. Biol. Evol. 24: 1596-1615.

van Rie J., McGaughey W.H., Johnson D.E., Barnett B.D., Van Mellaert H. (1990) Mechanism of Insect Resistance to 
the Microbial Insecticide Bacillus thuringiensis. Science 247: 72-74.

Walters F.S., Slatin S.L., Kuleszca C.A., English L.H. (1993) Ion Channel activity of $N$-terminal fragments from CryIA(c) $\delta$-endotoxin. Biochem. Biophys. Res. Commun. 196: 921-926.

Wiederstein M., Sippl M.J. (2007) ProSA-web: interactive web service for the recognition of errors in three dimensional structures of proteins. Nucleic Acids Res. 35: W407-410.
Wu D., Aronson A.I. (1992) Localized mutagenesis defines regions of the Bacillus thuringiensis delta-endotoxin involved in toxicity and specificity. Proc. Natl. Acad. Sci. 86: 4037-4041.

Xia L.Q., Zhao X.M., Ding X.Z., Wang F.X., Sun Y.J. (2008) The theoretical $3 D$ structure of Bacillus thuringiensis Cry5Ba. J. Mol. Model. 14: 843-848. 